\title{
Bromine-lithium exchange on gem-dibromoalkenes \\ Part 1: batch vs microflow conditions
}

Baptiste Picard, ${ }^{\mathrm{a}}$ Katia Perez, ${ }^{\mathrm{a}, \mathrm{b}}$ Thomas Lebleu, ${ }^{\mathrm{a}}$ Daniela Vuluga, ${ }^{\mathrm{b}}$ Fabrice Burel, ${ }^{\mathrm{b}}$ David C. Harrowven, ${ }^{\mathrm{c}}$ Isabelle Chataigner, ${ }^{\mathrm{a}}$ Jacques Maddaluno, ${ }^{\mathrm{a}}$ and Julien Legros ${ }^{\mathrm{a},{ }^{*}}$

a Normandie Univ, INSA Rouen, UNIROUEN, CNRS, COBRA, 76000 Rouen, France. Email: julien.legros@univ-rouen.fr

b Normandie Univ, INSA Rouen, UNIROUEN, CNRS, PBS, 76000 Rouen, France.

c Chemistry, University of Southampton, Highfield, Southampton, UK

This article is dedicated to the memory of Prof. Jun-ichi Yoshida

\section{Graphical abstract}

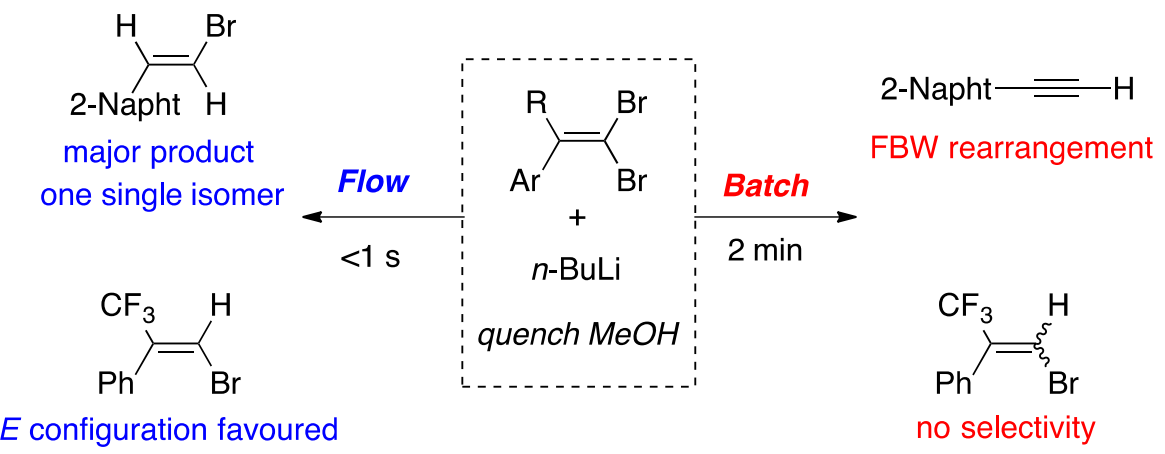

\begin{abstract}
The generation and reactivity of two model vinyl carbenoids from gem-dibromoalkenes $\mathbf{1}$ were studied in microflow systems. From substrate $\mathbf{1 a}\left(\mathrm{R}=\mathrm{Ph}, \mathrm{CF}_{3}\right)$, the lithium-bromine exchange could be simply performed within $30 \mathrm{~ms}$ at $20{ }^{\circ} \mathrm{C}$ with very good $E$-selectivity whereas the reaction was unselective under batch conditions, even at $-78{ }^{\circ} \mathrm{C}$. Moreover, the unstable carbenoid generated from $\mathbf{1 b}(\mathrm{R}=\mathrm{Ph}, \mathrm{H})$ could be trapped as the major product while only the Fritsch-Buttemberg-Wiechell rearrangement product was obtained in a flask under cryogenic conditions.
\end{abstract}

Keywords Fritsch-Buttemberg-Wiechell rearrangement, carbenoid, organolithium compounds, selectivity, microfluidics. 


\section{Introduction}

Carbenoids are defined as compounds whose reactivity is similar to that of carbenes, but which do not have the very particular electronic configuration of the latter. ${ }^{1}$ In the triplet state, carbenes have two equal, unmatched electrons in two distinct orbitals. In the singlet state, generally less stable, both electrons share the same orbital, leaving a second, vacant orbital on the same carbon atom. In contrast, carbenoids have an electronic distribution reminiscent of the singlet state of carbenes. Indeed, a carbenoid carbon atom will be substituted in such a way as to undergo both an accepting inductive effect and a powerful donor inductive effect from its substituents. Because of the large variety of possible substituents, carbenoids can display a dramatic change in behavior and reactivity. Common carbenoids are those bearing both an electronegative heteroatom and an alkali or alkaline earth metal (more rarely a transition metal). In this case, as a rule of the thumb, metal electropositivity correlates with the carbenoid reactivity, and the lability of the electronegative group further enhances this trend. As such, bromo-lithiated carbenoids ${ }^{2}$ are mostly unstable and vinyl carbenoids 2 obtained by lithium-halogen exchange from 1 may undergo fast Fritsch-Buttemberg-Wiechell (FBW) rearrangement into alkynes $3 .^{1}$ This ability to undergo FBW rearrangement is highly dependent on the nature of the electronegative substituent, the metal and the migrating group R. Vinyl carbenoids bearing aryl groups, or an arene and a hydrogen atom, are particularly susceptible to this transformation (Scheme 1).

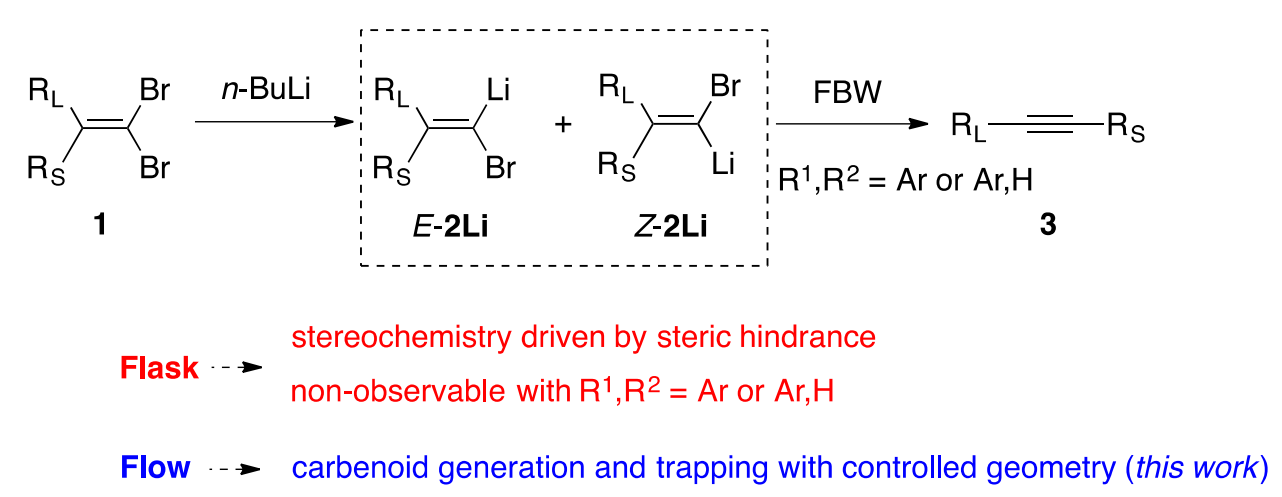

Scheme 1 Synthesis and reactivity of vinylcarbenoids $\mathbf{2}$ from gem-dibromoalkenes 1

In the case of stable carbenoids from gem-dibromovinyl systems, ${ }^{3}$ the metal-halogen exchange is often driven by steric decompression (Scheme 1 ), ${ }^{4}$ though direct metalation can be favored with a metal-chelating group (e.g. $\mathrm{R}=\mathrm{MOM}){ }^{5}$ Carbenoids of this type have occasionally been used in synthesis, ${ }^{5,6}$ though most lithium/bromine vinyl carbenoids 2 are used in FBW rearrangements (Corey-Fuchs protocol) and knowledge of their properties 
remains fragmentary. The use of vinyl carbenoids 2 would provide a means to quickly build a trisubstituted alkene bearing a bromine atom and with a defined configuration, opening up many synthetic possibilities. In this work we show how microflow conditions allow: 1) better control of regioselectivity when there is no clear discriminating R group, and 2) trapping of the carbenoid intermediates $\mathbf{2}$ before the fast FBW rearrangement or decomposition occurs.

\section{Results and discussion}

Microflow technology has been shown to be an exceptional tool to circumvent chemical selectivity issues related to competitive consecutive reactions. ${ }^{7-9}$ By virtue of excellent heat and mass transfer, and the precise control of reaction time in microreactors (residence time $t^{R}$ ), highly reactive compounds can be generated and intercepted in such systems before further adverse transformation can occur. This "flash chemistry" concept was introduced by Yoshida and over the last 10 years has been exploited successfully with short-lived organolithium intermediates ( $<1$ second).$^{10-14}$ The speed of bromine-lithium exchange and the propensity of some vinyl carbenoids 2 to undergo FBW rearrangement make microfluidics a useful tool to perform reaction. For this study, we chose two gem-dibromoalkenes as model substrates, bearing respectively $\mathrm{CF}_{3} /$ phenyl groups (1a) and hydrogen atom/naphthyl substituents (1b), as their behavior has been reported in the literature. ${ }^{15,16} \mathrm{Gem}$ dibromoalkenes 1 were treated with $n$-butyllithium in THF and HMPA at $-78{ }^{\circ} \mathrm{C}$ in a flask and quenched with methanol after 2 minutes. Under these conditions, 1a smoothly afforded the monobromo-olefine $\mathbf{2} \mathbf{a H}$ (full conversion), resulting from the trapping of a proton by the intermediate carbenoid $\mathbf{2 a L i}$ which is stable at this temperature. ${ }^{15}$ However, the low discrimination between the $\mathrm{CF}_{3}$ and phenyl group resulted in poor selectivity in the lithiumbromine exchange leading to a 55:45 E/Z ratio (Scheme 2). Stereochemical assignment was confirmed by bidimensional heteroNOESY ${ }^{1} \mathrm{H}-{ }^{19} \mathrm{~F}$ and seems in accordance with the literature. ${ }^{17}$ In contrast, under the same conditions substrate $\mathbf{1 b}$, bearing a substituent with a higher aptitude for migration, underwent rearrangement to naphthylacetylene $\mathbf{3 b}$, with only traces of the carbenoid interception product (Scheme 2). ${ }^{16}$ Notably, this result displayed significant variation upon repetition, and sometimes a small quantity of $\mathbf{2} \mathbf{b H}$ and/or starting material $\mathbf{1 b}$ could be observed, though $\mathbf{3 b}$ was observed as the major product in all experiments (down to 74\%, 5 experiments). 


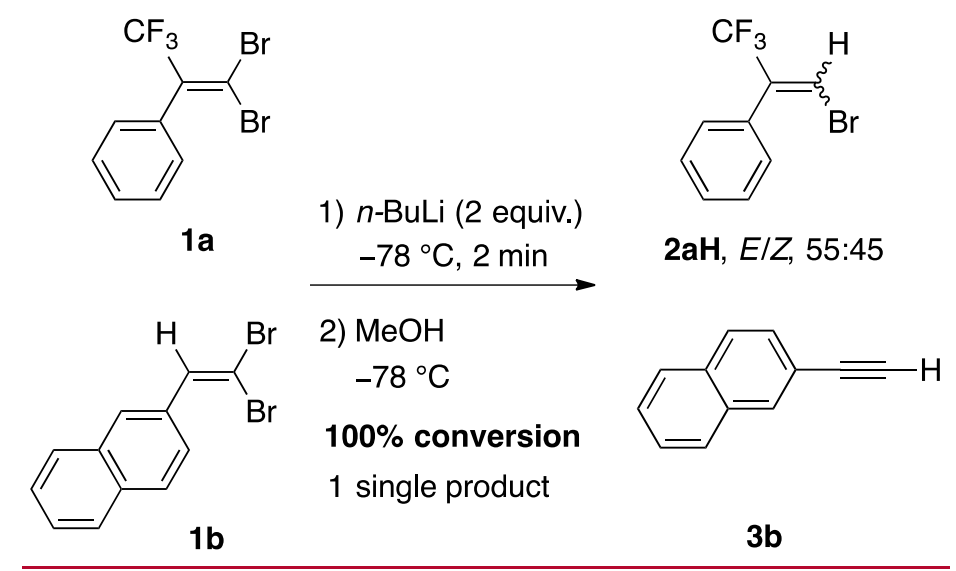

Scheme 2 Reaction of gem-dibromoalkenes $\mathbf{1 a}$ and $\mathbf{1 b}$ with $n$-BuLi under cryogenic batch conditions

These experiments confirm that under cryogenic batch conditions and short reaction times, no regioselectivity can be observed in the obtained product 2aH. For the hydrogensubstituted carbenoid generated from $\mathbf{1 b}$, the FBW rearrangement is so fast that it evades interception as a reaction intermediate. Furthermore, these batch reactions lacked reproducibility for $\mathbf{1 b}$ lithiation, prompting us to find more suitable operating conditions.

Our investigations under microflow conditions began with a system consisting of 2 inlets (dibromoalkene 1a in THF, $n$-BuLi in hexanes) reacting in a microreactor with a third inlet feeding methanol (30\% solution in THF) as a quench for the reaction. Results are reported in Table 1. In a short microreactor consisting of two T-shaped stainless steel micromixers $(\mathrm{V}=0.57 \mu \mathrm{L}, \mathrm{ID}=0.5 \mathrm{~mm})$ and a reactor tube $(\mathrm{L}=35 \mathrm{~mm}$; $\mathrm{ID}=0.5,0.762$ or 1 $\mathrm{mm}), n$-BuLi (0.4 M, 2 equiv.) and 1a were introduced. Our initial experiment was conducted under similar conditions to those conducted in batch. With HMPA as an additive under cryogenic conditions $\left(-78{ }^{\circ} \mathrm{C}\right)$ and $t^{R}=80 \mathrm{~ms}$, full conversion to $\mathbf{2 a H}$ was observed with complete selectivity for the $E$ isomer (entry 1). Interestingly, the same result was attained when the reaction was conducted at $0{ }^{\circ} \mathrm{C}$. For entries 1 and 2 pre-cooling loops ( $1 \mathrm{~m}$ length) were employed to set the temperature of the incoming reagents. ${ }^{10}$ One drawback with their use is the dramatic increase in the dead volume of the flow system leading to significant loss of material, higher pressure drops and more complex reaction setups. Therefore, we attempted to perform this reaction in a temperature-controlled bath at $20{ }^{\circ} \mathrm{C}$. At this unusually high temperature for a $\mathrm{Br}-\mathrm{Li}$ exchange, conversion and selectivity for the $E$ isomer were $>99 \%$ (entry 3).

We next examined the reaction without HMPA as its mutagenicity restricts its applications in synthesis. We found that reaction was successful at $20^{\circ} \mathrm{C}(>99 \%$ conversion toward $2 \mathbf{a H}$ ), even in a larger microreactor (ID $=1 \mathrm{~mm}, t^{R}=220 \mathrm{~ms}$ ), but $E / Z$ selectivity 
reduced to 61:39 (entry 4). With a smaller reactor $(I D=0.5 \mathrm{~mm}$ ) the isomeric ratio was enhanced to 81:19 with $t^{R}=50 \mathrm{~ms}$ (entry 5), and to 88:12 with a shorter reaction time $\left(t^{R}=50\right.$ ms, entry 6).

Table 1 Generation and trapping of carbenoid from 1a under microflow conditions

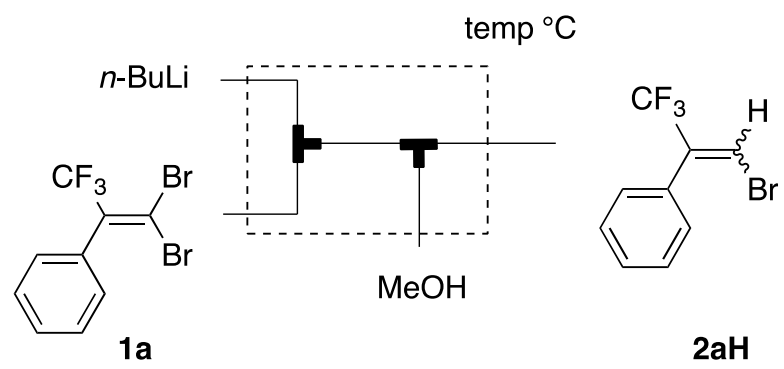

\begin{tabular}{llllllll}
\hline Entry & Total flow rate $(\mathrm{mL} / \mathrm{min})$ & Temp. $\left({ }^{\circ} \mathrm{C}\right)$ & Additive & $n$-BuLi (eq.) & $t^{R}(\mathrm{~s})$ & 2aH (\%) & E/Z (\%) \\
\hline 1 & 4 & -78 & HMPA & 2 & $0.08^{\mathrm{a}}$ & $>99$ & $>99: 1$ \\
2 & 4 & 0 & HMPA & 2 & $0.08^{\mathrm{a}}$ & $>99$ & $>99: 1$ \\
3 & 4 & 20 & HMPA & 2 & $0.08^{\mathrm{a}}$ & $>99$ & $>99: 1$ \\
4 & 7.5 & 20 & - & 2 & $0.22^{\mathrm{b}}$ & 71 & $61: 39$ \\
5 & 7.5 & 20 & - & 2 & $0.05^{\mathrm{c}}$ & $>99$ & $81: 19$ \\
6 & 9.4 & 20 & - & 2 & $0.04^{\mathrm{c}}$ & $>99$ & $88: 12$ \\
\hline
\end{tabular}

${ }^{\mathrm{a}} 0.762 \mathrm{~mm}$ ID tubing. ${ }^{\mathrm{b}} 1 \mathrm{~mm}$ ID tubing. ${ }^{\mathrm{c}} 0.5 \mathrm{~mm}$ ID tubing. Yield and selectivity determined by GC.

Therefore, under flash conditions in a microflow device at room temperature full conversion to $\mathbf{2} \mathbf{a H}$ can be achieved in the absence of HMPA, albeit with a reduction in $E / Z$ selectivity. As a comparison, under batch conditions at room temperature, even on small scale (e.g. $0.5 \mathrm{mmol}$ of 1a in THF ( $5 \mathrm{~mL}$ ) in a $25 \mathrm{~mL}$ flask immersed in a water bath at $20{ }^{\circ} \mathrm{C}$ ), the addition of $n$-BuLi (2.6 M, $1 \mathrm{mmol})$ proved so exothermic that the THF immediately boiled and a complex product mixture was given. In stark contrast, the use of a microflow reactor kept the exothermicity in check without using expensive cryogenic conditions. To our knowledge, this is the first example of such a reaction being performed successfully at room temperature. Interestingly, selectivity at $20^{\circ} \mathrm{C}$ in flow was higher than at $-78{ }^{\circ} \mathrm{C}$ under batch conditions. This suggests that the reaction course relies on the quality of mixing (ie reactor shape and flow rate) and that this in turn effects the aggregation state of organolithium species and their reactivity. Indeed, $n$-BuLi is introduced as a hexane solution whereas $\mathbf{1 a}$ is solubilized in THF (for reasons of stability, $n$-BuLi cannot be used as a solution in ether solutions, ${ }^{20}$ and a hexane solution of $\mathbf{1 a}$ caused immediate clogging of the system). 
Following, the study of $\mathrm{Br} / \mathrm{Li}$ exchange on the stable vinyl carbenoid $\mathbf{2 a L i}$, our investigation next examine the reactivity of the carbenoid $\mathbf{2} \mathbf{b L} \mathbf{i}$, generated from $\mathbf{1 b}$, towards trapping and the competitive FBW reaction, respectively leading to $\mathbf{2} \mathbf{b} \mathbf{H}$ and $\mathbf{3 b}$ (Table 2).

Table 2 Generation and trapping of carbenoid from $\mathbf{1 b}$ under microflow conditions. ${ }^{\text {a }}$

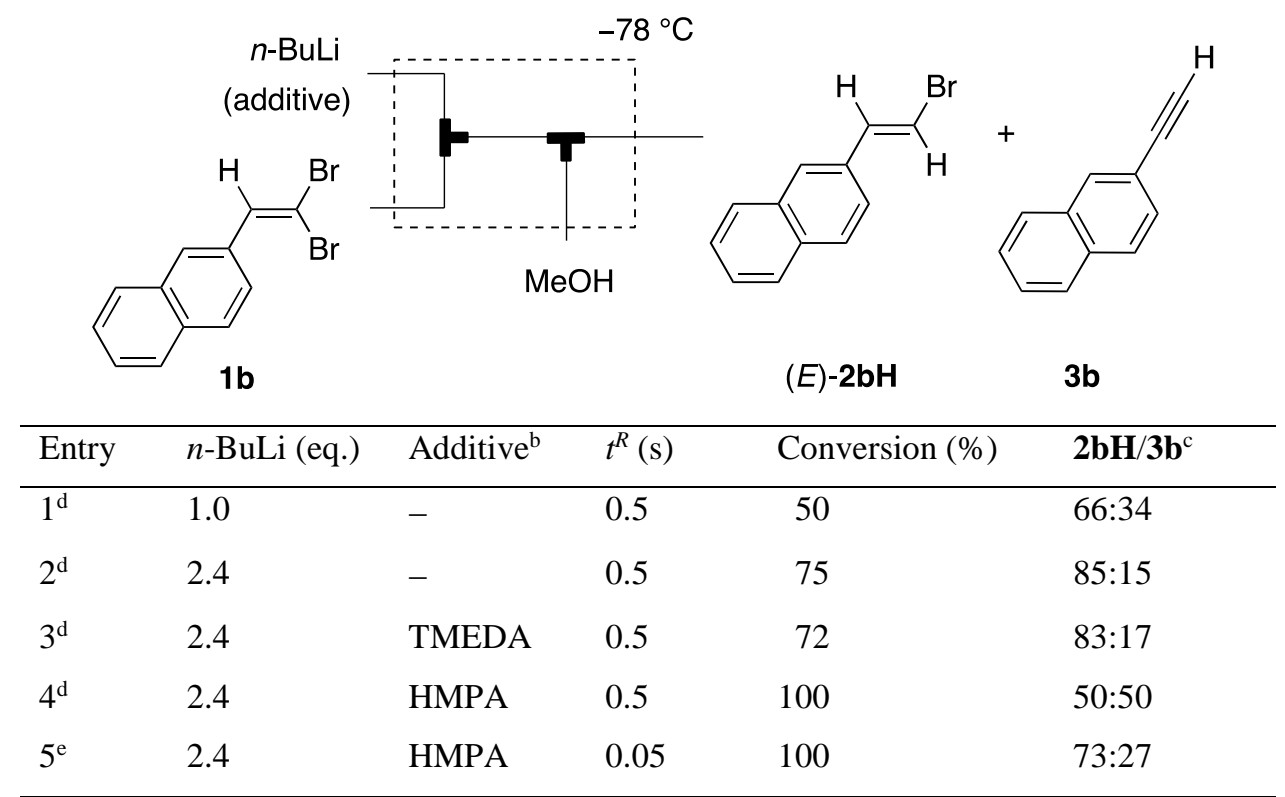

${ }^{\text {a }}$ Flow rates: $n$-BuLi (in hexane) $=5 \mathrm{~mL} / \mathrm{min} ; \mathbf{1 b}$ (in THF) $=10 \mathrm{~mL} / \mathrm{min} ; \mathrm{MeOH}=10$ $\mathrm{mL} / \mathrm{min} .{ }^{\mathrm{b}}$ Only $(E)$ isomer was obtained. ${ }^{\mathrm{c}}$ Ratio additive/ $n$-BuLi 1:1. Additive was added at the last minute at $0{ }^{\circ} \mathrm{C} .{ }^{\mathrm{d}} \mathrm{V}$ microreactor $=132 \mu \mathrm{L}(\mathrm{ID}=0.762 \mathrm{~mm}) .{ }^{\mathrm{e}} \mathrm{V}$ microreactor $=12.5 \mu \mathrm{L}(\mathrm{ID}=0.762 \mathrm{~mm})$. Yield and selectivity determined by GC.

Experiments were conducted in a microflow system similar to that used in the aforementioned study with syringe pumps for the introduction of $n$-BuLi (various concentrations in hexane), substrate $\mathbf{1 b}$ (in THF) and methanol using stainless steel tubing and T-shaped micromixers $(\mathrm{V}=0.57 \mu \mathrm{L}$, ID $=0.5 \mathrm{~mm})$. The microreactor $(\mathrm{V}=132 \mu \mathrm{L})$ was immersed in a temperature-controlled bath at $-78{ }^{\circ} \mathrm{C}$. The first experiment was performed in $0.5 \mathrm{~s}$ with 1 equiv. $n$-BuLi then quenched with methanol at the third inlet (entry 1 ). Conversion of the substrate was modest (50\%) with (E)-2bH and the FBW alkyne $\mathbf{3 b}$ formed in a 2:1 ratio. Due to the large difference in size between the substituents of $\mathbf{1 b}(\mathrm{H} v \mathrm{~s}$ naphthyl), the Br-Li exchange took place to afford the highest decompression and therefore gave the $(E)$-vinylbromide selectively. To increase the rate of the halogen-lithium exchange, the quantity of $n$-BuLi was increased to 2.4 equiv. (double concentration of $n$-BuLi with the same flow rates as entry 1). In this case, both conversion (75\%) and selectivity increased (2bH/3b, 85:15; entry 2). The influence of additives (1 equivalent with respect to $n$-BuLi in the same syringe) was then assessed (entries 3-5). Notably, conversion and product ratio were 
similar when TMEDA was added (entry 3), in contrast to reactions in the presence of HMPA which gave full conversion to $\mathbf{2} \mathbf{b H}$ and $\mathbf{3 b}$ at the expense of selectivity ( 1:1, entry 4). Notably, in a smaller microreactor $(12.5 \mu \mathrm{L})$ with a residence time of $50 \mathrm{~ms}$ (entry 5), full conversion could be achieved leading to a $73 \%$ yield of the desired product $\mathbf{2} \mathbf{b H}$, with only $27 \%$ of the FBW product $\mathbf{3 b}$.

\section{Conclusion}

In conclusion, we have been able to generate vinyl carbenoids in microflow systems through lithium-halogen exchange on two gem-dibromoalkenes. Under these flash chemistry conditions we realised outcome that could not be achieved under classical conditions. Thus, from dibromide 1a, lithium-bromine exchange could be performed in microflow at $20{ }^{\circ} \mathrm{C}$ in $30 \mathrm{~ms}$ and led to high $E$-selectivity in stark contrast to the irreproducible results attained under batch conditions. Similarly, the unstable carbenoid generated from $\mathbf{1 b}$ could be trapped with methanol to produce $(E)-\mathbf{2 b H}$ as major product when the Fritsch-Buttemberg-Wiechell rearrangement product $\mathbf{3 b}$ was the sole product observed when the reaction was conventionally performed in a round-bottomed flask under cryogenic conditions. It is however much likely that the specific features of the microflow reactor (flow rate, shape) influence the degree of aggregation of organolithium compounds, potentially impacting reaction course and selectivity. We are currently examining this hypothesis more fully and will be reporting our findings in due course.

On behalf of all authors, the corresponding author states that there is no conflict of interest.

\section{Acknowledgements}

The authors gratefully acknowledge the European France-(Manche)-England cross-border cooperation program INTERREG IV A “AI-CHEM CHANNEL” and INTERREG V A "LABFACT", co-financed by ERDF, for financial support. The authors from Normandie Université also thank the Tremplin Carnot I2C, the Labex SYNORG (ANR-11-LABX-0029) and the Région Normandie. K.P. is grateful to Ministro de Educación Superior, Ciencia y Tecnología of the Dominican Republic for fellowship (CALLIOPE programme). J.L. is grateful to Dr M. De Paolis and Prof. H. Oulyadi for fruitful discussion and assistance.

\section{References}

(1) Knorr, R. Alkylidenecarbenes, Alkylidenecarbenoids, $\uparrow$ and Competing Species: 
Which Is Responsible for Vinylic Nucleophilic Substitution, [1 + 2] Cycloadditions, 1,5-CH Insertions, and the Fritsch-Buttenberg-Wiechell Rearrangement? Chem. Rev. 2004, 104 (9), 3795-3850. https://doi.org/10.1021/cr030616h.

(2) Capriati, V.; Florio, S. Anatomy of Long-Lasting Love Affairs with Lithium Carbenoids: Past and Present Status and Future Prospects. Chemistry - A European Journal 2010, 16 (14), 4152-4162. https://doi.org/10.1002/chem.200902870.

(3) Chelucci, G. Synthesis and Metal-Catalyzed Reactions of Gem-Dihalovinyl Systems. Chem. Rev. 2012, 112 (3), 1344-1462. https://doi.org/10.1021/cr200165q.

(4) Harada, T.; Katsuhira, T.; Hattori, K.; Oku, A. Stereochemistry in Carbenoid Formation by Bromine/Lithium and Bromine/Zinc Exchange Reactions of Gem-Dibromo Compounds. Tetrahedron 1994, 50 (27), 7987-8002. https://doi.org/10.1016/S00404020(01)85284-4.

(5) Fürst, R.; Rinner, U. Synthesis of an Advanced Intermediate of the Jatrophane Diterpene Pl-4: A Dibromide Coupling Approach. J. Org. Chem. 2013, 78 (17), 8748-8758. https://doi.org/10.1021/jo401480t.

(6) Mizuta, S.; Otaki, H.; Kitamura, K.; Nishi, K.; Watanabe, K.; Makau, J. N.; Hashimoto, R.; Usui, T.; Chiba, K. 3,3-Dibromo-2-Trifluoromethyl Acrylic Acid Ethyl Ester: A Versatile Platform for the Stereoselective Preparation of Functionalized- $\alpha$-Trifluoromethyl $\alpha, \beta$-Unsaturated Lactones and Trifluoromethyl Pyrazolinones. Org. Chem. Front. 2016, 3 (12), 1661-1667. https://doi.org/10.1039/C6QO00360E.

(7) Wirth, T. Novel Organic Synthesis through Ultrafast Chemistry. Angew. Chem. Int. Ed. 2017, 56 (3), 682-684. https://doi.org/10.1002/anie.201609595.

(8) Yoshida, J. Flash Chemistry: Fast Organic Synthesis in Microsystems; Wiley: Hoboken, N.J, 2008.

(9) Yoshida, J.; Kim, H.; Nagaki, A. "Impossible” Chemistries Based on Flow and Micro. J Flow Chem 2017, 7 (3), 60-64. https://doi.org/10.1556/1846.2017.00017.

(10) Usutani, H.; Tomida, Y.; Nagaki, A.; Okamoto, H.; Nokami, T.; Yoshida, J. Generation and Reactions of O-Bromophenyllithium without Benzyne Formation Using a Microreactor. J. Am. Chem. Soc. 2007, 129 (11), 3046-3047. https://doi.org/10.1021/ja068330s.

(11) Nagaki, A.; Matsuo, C.; Kim, S.; Saito, K.; Miyazaki, A.; Yoshida, J. Lithiation of 1,2-Dichloroethene in Flow Microreactors: Versatile Synthesis of Alkenes and Alkynes by Precise Residence-Time Control. Angewandte Chemie International Edition 2012, 51 (13), 3245-3248. https://doi.org/10.1002/anie.201108932.

(12) Kim, H.; Nagaki, A.; Yoshida, J. A Flow-Microreactor Approach to Protecting-GroupFree Synthesis Using Organolithium Compounds. Nature Communications 2011, 2, 264. https://doi.org/10.1038/ncomms1264.

(13) Nagaki, A.; Ichinari, D.; Yoshida, J. Three-Component Coupling Based on Flash Chemistry. Carbolithiation of Benzyne with Functionalized Aryllithiums Followed by Reactions with Electrophiles. J. Am. Chem. Soc. 2014, 136 (35), 12245-12248. https://doi.org/10.1021/ja5071762.

(14) Kim, H.; Min, K.-I.; Inoue, K.; Im, D. J.; Kim, D.-P.; Yoshida, J. Submillisecond Organic Synthesis: Outpacing Fries Rearrangement through Microfluidic Rapid Mixing. Science 2016, 352 (6286), 691-694. https://doi.org/10.1126/science.aaf1389.

(15) Sam, B.; Montgomery, T. P.; Krische, M. J. Ruthenium Catalyzed Reductive Coupling of Paraformaldehyde to Trifluoromethyl Allenes: CF3-Bearing All-Carbon Quaternary Centers. Org. Lett. 2013, 15 (14), 3790-3793. https://doi.org/10.1021/ol401771a.

(16) Khan, Z. A.; Wirth, T. Synthesis of Indene Derivatives via Electrophilic Cyclization. Org. Lett. 2009, 11 (1), 229-231. https://doi.org/10.1021/ol8024956.

(17) Morken, P. A.; Bachand, P. C.; Swenson, D. C.; Burton, D. J. Synthesis of Fluorinated 
1,2,3-Butatrienes from .Alpha.-Halovinyl Organometallic Reagents. J. Am. Chem. Soc. 1993, 115 (13), 5430-5439. https://doi.org/10.1021/ja00066a011.

(18) Hamdoun, G.; Sebban, M.; Cossoul, E.; Harrison-Marchand, A.; Maddaluno, J.;

Oulyadi, H. ${ }^{1}$ H Pure Shift DOSY: A Handy Tool to Evaluate the Aggregation and Solvation of Organolithium Derivatives. Chem. Commun. 2014, 50 (31), 4073-4075.

https://doi.org/10.1039/C4CC00111G.

(19) Reich, H. J. Role of Organolithium Aggregates and Mixed Aggregates in Organolithium Mechanisms. Chem. Rev. 2013, 113 (9), 7130-7178.

https://doi.org/10.1021/cr400187u.

(20) Stanetty, P.; Mihovilovic, M. D. Half-Lives of Organolithium Reagents in Common Ethereal Solvents. J. Org. Chem. 1997, 62 (5), 1514-1515. https://doi.org/10.1021/jo961701a. 